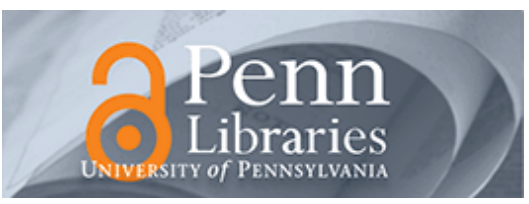

University of Pennsylvania

ScholarlyCommons

$1-27-2008$

\title{
A coalitional game model for spectrum pooling in wireless data access networks
}

\author{
Saswati Sarkar \\ University of Pennsylvania, swati@seas.upenn.edu \\ Chandramani Singh \\ Indian Institute of Science, Bangalore \\ Anurag Kumar \\ Indian Institute of Science, Bangalore
}

Follow this and additional works at: https://repository.upenn.edu/ese_papers

\section{Recommended Citation}

Saswati Sarkar, Chandramani Singh, and Anurag Kumar, "A coalitional game model for spectrum pooling in wireless data access networks", . January 2008.

Copyright 2008 IEEE. Reprinted from:

Sarkar, S.; Singh, C.; Kumar, A., "A coalitional game model for spectrum pooling in wireless data access networks," Information Theory and Applications Workshop, 2008 , vol., no., pp.310-319, Jan. 27 2008-Feb. 12008

URL: http://ieeexplore.iee.. rg/stamp/stamp.jsp?arnumber=4601066\&isnumber=4601007

This material is posted here with permission of the IEEE. Such permission of the IEEE does not in any way imply IEEE endorsement of any of the University of Pennsylvania's products or services. Internal or personal use of this material is permitted. However, permission to reprint/republish this material for advertising or promotional purposes or for creating new collective works for resale or redistribution must be obtained from the IEEE by writing to pubs-permissions@ieee.org. By choosing to view this document, you agree to all provisions of the copyright laws protecting it.

This paper is posted at ScholarlyCommons. https://repository.upenn.edu/ese_papers/490

For more information, please contact repository@pobox.upenn.edu. 


\title{
A coalitional game model for spectrum pooling in wireless data access networks
}

\begin{abstract}
We consider a setting in which several operators offer downlink wireless data access services in a certain geographical region. Each operator deploys several base stations or access points, and registers some subscribers. In such a situation, if operators pool their infrastructure, and permit the possibility of subscribers being served by any of the cooperating operators, then there can be overall better user satisfaction, and increased operator revenue. We use coalitional game theory to investigate such resource pooling and cooperation between operators. We use utility functions to model user satisfaction, and show that the resulting coalitional game has the property that if all operators cooperate (i.e., form a grand coalition) then there is an operating point that maximizes the sum utility over the operators while providing the operators revenues such that no subset of operators has an incentive to break away from the coalition. We investigate whether such operating points can result in utility unfairness between users of the various operators. We also study other revenue sharing concepts, namely, the nucleolus and the Shapely value. Such investigations throw light on criteria for operators to accept or reject subscribers, based on the service level agreements proposed by them. We also investigate the situation in which only certain subsets of operators may be willing to cooperate.
\end{abstract}

\section{Keywords}

coalitional game model, geographical region, revenue sharing concept, service level agreement, spectrum pooling, user satisfaction, utility function, wireless data access network, game theory, radio access networks

\section{Comments}

Copyright 2008 IEEE. Reprinted from:

Sarkar, S.; Singh, C.; Kumar, A., "A coalitional game model for spectrum pooling in wireless data access networks," Information Theory and Applications Workshop, 2008 , vol., no., pp.310-319, Jan. 27 2008-Feb. 12008

URL: http://ieeexplore.ieee.org/stamp/stamp.jsp?arnumber=4601066\&isnumber=4601007

This material is posted here with permission of the IEEE. Such permission of the IEEE does not in any way imply IEEE endorsement of any of the University of Pennsylvania's products or services. Internal or personal use of this material is permitted. However, permission to reprint/republish this material for advertising or promotional purposes or for creating new collective works for resale or redistribution must be obtained from the IEEE by writing to pubs-permissions@ieee.org. By choosing to view this document, you agree to all provisions of the copyright laws protecting it. 


\section{A coalitional game model for spectrum pooling in wireless data access networks}

\author{
Saswati Sarkar \\ Department of Electrical and Systems Eng. \\ University of Pennsylvania \\ 200 S. 33rd Street, Philadelphia 19104, USA \\ Email: swati@seas.upenn.edu
}

\author{
Chandramani Singh \\ Anurag Kumar \\ Electrical Communications Eng. Electrical Communications Eng. \\ Indian Institute of Science \\ Indian Institute of Science \\ Bangalore, 560012, India \\ Bangalore, 560012, India \\ Email: chandra@ece.iisc.ernet.in Email: anurag@ece.iisc.ernet.in
}

\begin{abstract}
We consider a setting in which several operators offer downlink wireless data access services in a certain geographical region. Each operator deploys several base stations or access points, and registers some subscribers. In such a situation, if operators pool their infrastructure, and permit the possibility of subscribers being served by any of the cooperating operators, then there can be overall better user satisfaction, and increased operator revenue. We use coalitional game theory to investigate such resource pooling and cooperation between operators. We use utility functions to model user satisfaction, and show that the resulting coalitional game has the property that if all operators cooperate (i.e., form a grand coalition) then there is an operating point that maximizes the sum utility over the operators while providing the operators revenues such that no subset of operators has an incentive to break away from the coalition. We investigate whether such operating points can result in utility unfairness between users of the various operators. We also study other revenue sharing concepts, namely, the nucleolus and the Shapely value. Such investigations throw light on criteria for operators to accept or reject subscribers, based on the service level agreements proposed by them. We also investigate the situation in which only certain subsets of operators may be willing to cooperate.
\end{abstract}

\section{INTRODUCTION}

The last decade has witnessed rapid proliferation of commercial wireless services. End users subscribe to the operators of their choice. Operators deploy service units like access points, base stations etc. in their coverage areas, and lease parts of the spectrum and allocate the corresponding frequency bands to the service units that they control. The service units in turn transmit and receive data, voice etc. in the allocated bands to and from the end users subscribing to the respective operators. An end user may experience poor quality of service owing to overload or poor channel conditions of the bands licensed by the operator it has subscribed to, and may not receive any service if it is outside the coverage area of its operator. For instance, small towns and rural areas in India are primarily covered by the national telecommunications operator, BSNL, whereas private operators like Airtel, Hutch provide better coverage in cities. If, however, different operators pool their resources, then the end users of one operator can be served by service units of another operator when they move outside the coverage areas of their own operator and also in the event of overload or sustained poor transmission quality. Such cooperation is likely to substantially enhance the aggregate user satisfaction, and thereby revenue, which can in turn be appropriately shared among the participating operators. Indeed such cooperation may be viewed as a primitive form of spectrum pooling [1] that requires no changes in the equipment; only the high level network control policies are changed.

The success of such cooperation is however contingent upon developing a rational basis for sharing the aggregate revenue among the cooperating entities. This is because an operator will cooperate with others not merely to improve the aggregate revenue, but only when such cooperation increases its share of revenue, and it perceives that its share of revenue is commensurate with its role in attaining the aggregate reward. Such a basis will allow an operator to optimize its revenue share by appropriately determining who it should cooperate with and how it should cooperate. Specifically, an operator's revenue should depend on the revenue fetched by the end users that subscribe to it and the revenue it generates for its and others' end users. Both of the above depend on (a) the locations of the end users, (b) the channel gains of the frequency bands, (c) service demands of the end users, (d) the ownership of the end users, (e) allocations of the end users to the service units, (f) locations of the service units, and $(\mathrm{g})$ which operators are willing to cooperate and (h) whether and how each operator distinguishes among the end users that belong to it and those that belong to other operators. Note that operators control (e), (f), (g), (h), and also (c) and (d) in part (by choosing not to accept end users with certain service demands), but does not control (a) and (b).

The contribution of this paper is to develop a framework 
that determines how the operators share the aggregate revenue commensurate with their resources and actions. We model cooperation among operators using the theory of coalitional games [2] (Section II). We consider a coalitional game among the operators, and obtain the above revenues as the solution of a concave optimization which depends on utilities or satisfaction functions of end users and resource utilization in presence of cooperation in the wireless context (specifically on factors (a) to (h) above). Our coalitional game model reveals that cooperation among operators can induce unfairness among end users. Specifically, depending on the utility functions of the end users, cooperation among carriers may decrease the quality of service experienced by some end users, although such cooperation enhances the aggregate quality of service. This may in turn reduce the customer base of the respective operator and thereby affect its share of the aggregate revenue. Thus, an operator must carefully assess the impact of cooperation on its customers, and judiciously determine the utility functions and/or ensure certain minimum quality of service to its end users during cooperation, even if this decreases the aggregate revenue.

We next prove that when all operators cooperate (i.e., forms a grand coalition), there exists a revenue allocation for the operators that ensures that if a subset of operators separate from the grand coalition then, irrespective of the manner in which the subset shares the aggregate revenue it generates after separating, at least one operator in the subset is worse off than in the grand coalition (Section III-A). Such a revenue allocation renders the grand coalition stable as no subset of operators now has an incentive to separate; this is socially desirable as the aggregate revenue is maximized when all operators cooperate. We also show that one such allocation can be computed from a solution of a convex optimization whose number of variables and constraints are polynomial in the number of operators, service units and end users. In coalitional game terminology, the set of all such allocations is said to be the core [2]. Thus, we have shown that the core is non-empty and have obtained an allocation in the core in polynomial time. This is interesting from a game theoretic point of view as there are several games that satisfy neither of these properties.

We next investigate other solution concepts that provide a basis for sharing the revenue, namely the nucleolus and the Shapley value [2] (Section III-B). The motivation for this investigation is that both the above exist uniquely for any coalitional game while the core consists of several allocations in general and also for the game we consider. We show that the nucleolus renders the grand coalition stable, but the Shapley value does not attain this goal.
The Shapley value therefore does not provide a desirable basis for sharing revenues in this case.

An interesting direction for future research is to investigate how each operator can optimize its actions so as to maximize its share of the revenue as provided by the above sharing mechanisms (e.g., nucleolus or other allocations in the core). As a first step towards that end, we investigate which end users an operator should accept (Section III-C). The question is important, as an end user will typically require a minimum quality of service guarantee (a service level agreement or a SLA) which an operator needs to honor if it accepts the end user, and such SLAs may reduce the aggregate revenue of the coalitions. But, an operator ought to accept an end user if its subscription enhances the revenue the operator earns, irrespective of how such an acceptance affects the aggregate revenue. We demonstrate that, although SLAs reduce the aggregate revenues of the coalitions, the ownership of the end users demanding the SLAs may increase the revenue the respective operators earn, in which case, the operators ought to accept the SLAs. Next, an end user may require a SLA, an operator can honor only when it cooperates with other operators. If the operator accepts such a SLA, it incurs a huge penalty when it is not cooperating with any other operator as then it cannot honor the SLA. Nevertheless, we show that there are instances where depending on (a) the service, the operator can offer to end users owned by other operators, and (b) the service, this specific end user can obtain from other operators, the presence of such an end user may actually enhance the operator's share of revenue. The operator should accordingly accept or reject such an end user.

Finally, all operators may not be willing to cooperate owing to factors other than revenue, e.g, lack of mutual trust etc. We model the trust relations using a graph, and using a graphical coalitional game, generalize our framework to accommodate such extraneous considerations (Section IV). We show that the grand coalition can be stabilized in this case as well. Thus, all operators that are allowed to cooperate can be induced to cooperate, which again is a socially desirable outcome.

We now comment on the related work in context of wireless networks. Game theoretic investigation of resource allocation in wireless networks, and spectrum allocation in particular, has so far largely relied on tools from non-cooperative game theory (e.g., [3], [4], [5], [6], [7]). Principles from cooperative game theory are likely to substantially enhance our understanding of this area, particularly since many of the resource allocation mechanisms rely on cooperation among nodes. Towards this end, Nash bargaining solutions have been proposed for power control and spectrum sharing among multiple 
users [8]. Coalitional games have recently been used for modeling cooperation among nodes in the physical layer [9], [10]. We propose a coalitional game model for a simple form of spectrum pooling in wireless data access networks. We model spectrum and service unit pooling among different operators. Thus, the specific coalitional games we consider, and the analytical results we obtain, significantly differ from those of the above papers.

\section{A COALITIONAL GAME FORMULATION}

Definition 2.1: A coalitional game with transferable payoff consists of a set of players $\mathcal{N}=1, \ldots, n$ and a characteristic function $v($.$) which is a real valued$ function on the power set of $\mathcal{N}$. For a subset $\mathcal{S}$ of $\mathcal{N}, v(\mathcal{S})$, the characteristic value of $\mathcal{S}$, provides the aggregate wealth generated by the players in $\mathcal{S}$, when only these players form a coalition, irrespective of the actions of the players in $\mathcal{N} \backslash \mathcal{S}$.

In this work we confine ourselves to coalitional games with transferable payoff, and hence omit the phrase transferable payoff in further discussion, without causing any ambiguity.

A coalitional game is fully defined once $\mathcal{N}$ and $v($. are specified. In this section, we formulate a coalitional game on the set of operators.

Let $\mathcal{N}=\{1, \ldots, n\}$ denote the set of operators. Each operator $i$ owns service units in the set $\mathcal{B}_{i}$ and end users (or simply users) in the set $\mathcal{M}_{i}$. We assume that a service unit and a user can be associated with only one operator, i.e., $\mathcal{B}_{i} \cap \mathcal{B}_{j}=\phi$ and $\mathcal{M}_{i} \cap \mathcal{M}_{j}=\phi$ for $i \neq j$. Let $\mathcal{B}$ and $\mathcal{M}$ be the sets of service units and users in the system respectively, and $|\mathcal{B}|=B,|\mathcal{M}|=M$. Let user $k$ needs a minimum rate $m_{k}$, which constitutes the service level agreement between $k$ and $k$ 's operator.

Each operator licenses certain frequency bands, which are allocated to the service units it owns. We assume that no two service units in a vicinity are assigned the same band, and a frequency band is licensed by at most one operator. Thus, the communications of different service units do not interfere. When service unit $j$ serves user $k, k$ receives a rate $R_{j k}$, which depends on the locations of $j, k$, channel gain of the frequency band used by $j$, transmission power and the reception gain of the two units. A service unit serves at most one user at a given time.

Definition 2.2: A coalition $\mathcal{S} \subseteq \mathcal{N}$ is a subset of operators who cooperate. For a coalition $\mathcal{S}, \mathcal{B}_{\mathcal{S}}, \mathcal{M}_{\mathcal{S}}$ are the sets of service units and users associated with operators in $\mathcal{S}$. The coalition $\mathcal{N}$ is termed as the grand coalition.

Let $\alpha_{j k}$ be the fraction of time, the service unit $j$ serves the user $k ;\left\{\alpha_{j k}, j \in \mathcal{B}_{\mathcal{S}}, k \in \mathcal{M}_{\mathcal{S}}\right\}$ are determined by the service allocation rules of the operators. A service unit $j$ may serve a user $k$, i.e., $\alpha_{j k}>0$, only when either both are associated with the same operator, or the operators associated with them are in a coalition. When user $k$ 's operator is part of the coalition $\mathcal{S}$, the rate obtained by $k$ is $y_{k}=\sum_{j \in \mathcal{B}_{\mathcal{S}}} \alpha_{j k} R_{j k}$. When users associated with operator $i$ receive rates $\mathbf{y}^{i}=\left\{y_{k}, k \in\right.$ $\left.\mathcal{M}_{i}\right\}$, then they generate a revenue of $U_{i}\left(\mathbf{y}^{i}\right)$, where $U_{i}($.$) is a concave increasing function. The function$ $U_{i}($.$) is the utility function of the operator i$, which reflects the aggregate satisfaction of the users associated with $i$. For example, utility functions can be linear, i.e., $U_{i}\left(\mathbf{y}^{i}\right)=\sum_{k \in \mathcal{M}_{i}} y_{k}$, or logarithmic, i.e., $U_{i}\left(\mathbf{y}^{i}\right)=$ $\sum_{k \in \mathcal{M}_{i}} \log \left(y_{k}\right)$.

Each operator can have constraints on the fraction of time its service units serve the users of other operators, which can be easily represented in the form $\mathbf{A} \boldsymbol{\alpha} \leq \mathbf{b}$. But such constraints may be superfluous in presence of the minimum rate constraints for the users. Thus we do not consider such constraints; nevertheless all our results hold in presence of such constraints.

Let $\mathcal{S} \subseteq \mathcal{N}$. Then, $v(\mathcal{S})$ is the maximum revenue generated by the operators in $\mathcal{S}$, when only the operators in $\mathcal{S}$ form a coalition. Note that the rates obtained, and hence the revenues generated by the users in $\mathcal{M}_{\mathcal{S}}$, do not depend on the actions of the operators in $\mathcal{N} \backslash \mathcal{S}$. Let $v(\phi)=0$.

P(S:- Maximize: $\sum_{i \in \mathcal{S}} U_{i}\left(\mathbf{y}^{i}\right)$

\section{Subject to:}

1) $\sum_{k \in \mathcal{M}_{\mathcal{S}}} \alpha_{j k} \leq 1, j \in \mathcal{B}_{\mathcal{S}}$,

2) $\sum_{j \in \mathcal{B}_{\mathcal{S}}} \alpha_{j k} \leq 1, k \in \mathcal{M}_{\mathcal{S}}$

3) $y_{k} \leq \sum_{j \in \mathcal{B}_{\mathcal{S}}} \alpha_{j k} R_{j k}, k \in \mathcal{M}_{\mathcal{S}}$,

4) $y_{k} \geq m_{k}, k \in \mathcal{M}_{\mathcal{S}}$

5) $\alpha_{j k} \geq 0, j \in \mathcal{B}_{\mathcal{S}}, k \in \mathcal{M}_{\mathcal{S}}$,

6) $y_{k} \geq 0, k \in \mathcal{M}_{\mathcal{S}}$

If $\mathbf{P}(\mathcal{S})$ is feasible, $v(\mathcal{S})$ equals the maximum value of the objective function of $\mathbf{P}(\mathcal{S})$, else $v(\mathcal{S})=-\infty$. Note that $\mathbf{P}(\mathcal{S})$ may be infeasible only when $m_{k}>0$ for some $k \in \mathcal{M}_{\mathcal{S}}$.

A coalitional game is superadditive if $v(\mathcal{S}) \geq$ $\sum_{i \in \mathcal{I}} v\left(\mathcal{S}_{i}\right)$ where $\left\{\mathcal{S}_{i}, i \in \mathcal{I}\right\}$ constitute a partition of $\mathcal{S}$. Here, if $\mathbf{P}(\mathcal{S})$ is infeasible, and $|\mathcal{S}|>1$, then $\mathbf{P}(\mathcal{T})$ is infeasible for some $\mathcal{T} \subset \mathcal{S}$. Next, if $\mathbf{P}(\mathcal{S})$ is feasible, $v(\mathcal{S}) \geq \sum_{i \in \mathcal{I}} v\left(\mathcal{S}_{i}\right)$ for any partition $\left\{\mathcal{S}_{i}, i \in \mathcal{I}\right\}$ of $\mathcal{S}$. Hence, the game we are considering, is superadditive. We assume that $v(\mathcal{N})$ is feasible, i.e., the grand coalition can satisfy all the minimum rate guarantees. Thus,

$$
v(\mathcal{N}) \geq \sum_{i \in \mathcal{I}} v\left(\mathcal{S}_{i}\right), \text { for any partition }\left\{\mathcal{S}_{i}, i \in \mathcal{I}\right\} \text { of } \mathcal{N}
$$


Thus, the aggregate revenue is maximized if all the users cooperate. Since the aggregate revenue is a measure of the user satisfaction, the aggregate user satisfaction is maximized when all the users cooperate.

We now elucidate $v($.$) using a simple example.$

Example 2.1: Let $n=2$, and each operator owns one service unit and two users. Let $R_{j k}=P$ if $k \in \mathcal{M}_{1}$, and $R_{j k}=G$ if $k \in \mathcal{M}_{2}$, for all $j \in \mathcal{B}$. Let $P<G$. Also, $m_{k}=0 \forall k \in \mathcal{M}$. Thus, $\mathbf{P}(\mathcal{S})$ is feasible for all $\mathcal{S} \subseteq \mathcal{N}$. First, let the utility functions be linear. Then, $v(\{1\})=P, v(\{2\})=G, v(\{1,2\})=2 G$. Thus, $v(\{1,2\})>v(\{1\})+v(\{2\})$. The optimum solution of $\mathbf{P}(\{1,2\})$ which attains $v(\{1,2\})$, assigns $\alpha_{j k}=0 \forall j \in$ $\mathcal{B}, k \in \mathcal{M}_{1}$. Thus, the users of operator 1 receive no service when the two operators cooperate. But, when operator 1 does not cooperate with operator 2 , an optimum solution of $\mathbf{P}(\{1\})$ assigns rates $\alpha P$ and $(1-\alpha) P$ to its users, where $0 \leq \alpha \leq 1$, i.e., both the users can potentially get non-zero rates. Thus, the coalition enhances aggregate revenue but induces unfairness for the users and may therefore reduce the customer base of individual operators. We later demonstrate that this may have serious repercussions on the revenues earned by individual operators (Section III-C).

Finally, note that the above unfairness can be mitigated by imposing appropriate minimum rate constraints for users, or by choosing different utility functions, both of which may in turn reduce the aggregate revenue. For example, when the minimum required rate $m_{k}=P / 2$ for all the users $k$, then $\mathbf{P}(\mathcal{S})$ is still feasible for all $\mathcal{S} \subseteq \mathcal{N}, v(\{1\})=P, v(\{2\})=G, v(\{1,2\})=$ $v(\{1\})+v(\{2\})=G+P<2 G$. Similarly, when $U_{i}\left(\mathbf{y}^{i}\right)=\sum_{k \in \mathcal{M}_{i}} \log \left(y_{k}\right), v(\{1\})=2 \log (P / 2)$, $v(\{2\})=2 \log (G / 2), v(\{1,2\})=v(\{1\})+v(\{2\})$. But, the optimum solutions of $\mathbf{P}(\{1,2\})$ and $\mathbf{P}(\{1\})$ (resp. $\mathbf{P}(\{2\})$ ) allocates rates $P / 2$ (resp. $G / 2$ ) to each of the users of operator 1 (resp. 2). Thus, in both of the above cases, the coalition does not enhance the aggregate revenue, reduces the revenue as compared to that with linear utility and no minimum rate constraints, but sustains the fair rate allocation among the users.

A coalitional game is convex if $v(\mathcal{S})+v(\mathcal{T}) \leq v(\mathcal{S} \cup$ $\mathcal{T})+v(\mathcal{S} \cap \mathcal{T})$ for all $\mathcal{S}, \mathcal{T} \subseteq \mathcal{N}$. The game we are considering, is not convex, as the following example shows.

Example 2.2: Let $n=3, \mathcal{B}_{i}=\{i\}, i=1,2,3, \mathcal{M}_{1}=$ $\phi, \mathcal{M}_{i}=\{i-1\}, i=2,3$. Let $R_{1 k}=G, k \in \mathcal{M}$ and $R_{j k}=P, j \in\{2,3\}, k \in \mathcal{M}$. Let $G>P$. Let $m_{k}=$ $0, k \in \mathcal{M}$. Let the utility functions be linear. Thus, $v(\{1\})=0, v(\{1,2\})=v(\{1,3\})=G, v(\{1,2,3\})=$ $G+P$. Let $\mathcal{S}=\{1,2\}, \mathcal{T}=\{1,3\}$. Then, $v(\mathcal{S})+v(\mathcal{T})=$ $2 G, v(\mathcal{S} \cup \mathcal{T})+v(\mathcal{S} \cap \mathcal{T})=G+P$. Thus, $v(\mathcal{S})+v(\mathcal{T})>$
$v(\mathcal{S} \cup \mathcal{T})+v(\mathcal{S} \cap \mathcal{T})$. Hence, this game is not convex.

\section{A FRAMEWORK FOR SHARING THE REVENUE GENERATED BY THE COALITIONS}

Definition 3.1: A revenue allocation $\mathbf{x}=\left(x_{1}, \ldots, x_{n}\right)$ for the operators is said to be an imputation if $\sum_{i=1}^{n} x_{i}=$ $v(\mathcal{N})$. The core, $\mathcal{C}$, is the set of imputations for which $v(\mathcal{S}) \leq \sum_{i \in \mathcal{S}} x_{i}$ for each $\mathcal{S} \subset \mathcal{N}$. Thus,

$$
\begin{aligned}
\mathcal{C}=\left\{\mathbf{x} \in \mathbb{R}^{n}:\right. & \sum_{i \in \mathcal{N}} x_{i}=v(\mathcal{N}), \\
& \left.\sum_{i \in \mathcal{S}} x_{i} \geq v(\mathcal{S}), \forall \mathcal{S} \subset \mathcal{N}\right\}
\end{aligned}
$$

In Example 2.1, $\mathcal{C}=\left\{\mathrm{x} \in \mathbb{R}^{2}: x_{1}+x_{2}=2 G, x_{1} \geq\right.$ $\left.P, x_{2} \geq G\right\}$.

Suppose $\mathcal{C}$ is non-empty. Let the operators form the grand coalition and share the aggregate revenue $v(\mathcal{N})$ as per an imputation $\mathbf{x} \in \mathcal{C}$. Next, let a subgroup $\mathcal{S} \subset \mathcal{N}$ of operators separates from the grand coalition and shares the aggregate revenue $v(\mathcal{S})$ as per $\mathbf{z}$. Now, if $z_{i} \leq x_{i}$ for an operator $i \in \mathcal{S}$, it will not have any incentive separating from the grand coalition. Thus, let $z_{i}>x_{i}$ for all $i \in \mathcal{S}$. Clearly, $\sum_{i \in \mathcal{S}} z_{i}=v(\mathcal{S})>\sum_{i \in \mathcal{S}} x_{i}$. This leads to a contradiction, since $\mathbf{x} \in \mathcal{C}$ (see 1 ). Thus, no subgroup will separate from the grand coalition. Thus, the grand coalition is stable, which is a socially desirable outcome, as the grand coalition maximizes the aggregate user satisfaction. This motivates the following definition.

Definition 3.2: An imputation stabilizes the grand coalition if it is in the core. The grand coalition is stabilizable if the core is non-empty.

The grand coalitions in several coalitional games cannot be stabilized (Example 260.3, p. 260, [2]), and it is in general NP-hard to determine whether the grand coalition in a given coalitional game can be stabilized [11]. Convexity of the game is a sufficient condition for the stabilizability of the grand coalition (p. 260, [2]), but we know that this sufficiency condition does not hold in our case (see Example 2.2).

Nevertheless, we next prove that, for the class of games we are considering, $\mathcal{C}$ is always non-empty, and subsequently we obtain an imputation $\mathrm{x} \in \mathcal{C}$.

\section{A. Is the grand coalition stabilizable?}

Using the specific structure of our game, and proof techniques presented in [12], [13], [14], [15], we prove that $\mathcal{C}$ is non-empty and hence the grand coalition can be stabilized (Section III-A). The proof is constructive in that it provides an imputation in $\mathcal{C}$ as well. 
Let $\boldsymbol{\lambda} \in \mathbb{R}^{\left|\mathcal{B}_{\mathcal{S}}\right|}, \boldsymbol{\beta}, \boldsymbol{\gamma}, \boldsymbol{\tau} \in \mathbb{R}^{\left|\mathcal{M}_{\mathcal{S}}\right|}$. Define

$$
f_{i}(\boldsymbol{\gamma}, \boldsymbol{\tau})=\max _{y_{k} \geq 0, k \in \mathcal{M}_{i}}\left(U_{i}\left(\mathbf{y}^{i}\right)-\sum_{k \in \mathcal{M}_{i}}\left(\gamma_{k}-\tau_{k}\right) y_{k}\right)
$$

D $(\mathcal{S})$ :- Minimize:

$\sum_{i \in \mathcal{S}}\left(f_{i}(\boldsymbol{\gamma}, \boldsymbol{\tau})+\sum_{j \in \mathcal{B}_{i}} \lambda_{j}+\sum_{k \in \mathcal{M}_{i}}\left(\beta_{k}-\tau_{k} m_{k}\right)\right)$

\section{Subject to:}

1) $\lambda_{j}+\beta_{k} \geq \gamma_{k} R_{j k}, j \in \mathcal{B}_{\mathcal{S}}, k \in \mathcal{M}_{\mathcal{S}}$,

2) $\lambda_{j} \geq 0, j \in \mathcal{B}_{\mathcal{S}}$

3) $\beta_{k} \geq 0, k \in \mathcal{M}_{\mathcal{S}}$

4) $\gamma_{k} \geq 0, k \in \mathcal{M}_{\mathcal{S}}$

5) $\tau_{k} \geq 0, k \in \mathcal{M}_{\mathcal{S}}$

Note that $\mathbf{D}(\mathcal{S})$ is the dual of $\mathbf{P}(\mathcal{S})$ for each $\mathcal{S} \subseteq \mathcal{N}$.

Formulate $\mathbf{D}(\mathcal{N})$ by appropriately defining vectors $\boldsymbol{\lambda}, \boldsymbol{\beta}, \boldsymbol{\gamma}$ and $\boldsymbol{\tau}$. Let $\mathcal{D}$ constitute the set of optimal solutions of $\mathbf{D}(\mathcal{N})$. Clearly, $\mathbf{D}(\mathcal{S})$ is feasible for each $\mathcal{S} \subseteq \mathcal{N}$. Thus, $\mathcal{D} \neq \phi$. Let

$$
\begin{aligned}
\mathcal{O}= & \left\{\mathbf{x}^{*} \in \mathbb{R}^{n}: x_{i}^{*}=f_{i}\left(\boldsymbol{\gamma}^{*}, \boldsymbol{\tau}^{*}\right)+\sum_{j \in \mathcal{B}_{i}} \lambda_{j}^{*}+\sum_{k \in \mathcal{M}_{i}}\right. \\
& \left.\left(\beta_{k}^{*}-\tau_{k}^{*} m_{k}\right) \text { for some }\left(\boldsymbol{\lambda}^{*}, \boldsymbol{\beta}^{*}, \boldsymbol{\gamma}^{*}, \boldsymbol{\tau}^{*}\right) \in \mathcal{D}\right\}
\end{aligned}
$$

Theorem 3.1: $\mathcal{O} \neq \phi$ and $\mathcal{O} \subseteq \mathcal{C}$.

Discussion: Note that, once we know that $\mathcal{C} \neq \phi$, an imputation in $\mathcal{C}$ can be obtained using (1). But, the computation time will be substantial for large $n$, as (1) characterizes $\mathcal{C}$ using $2^{n}$ inequalities. However, note that $f_{i}(\boldsymbol{\gamma}, \boldsymbol{\tau})$ is a convex function, as it is the pointwise maximum value of a family of affine functions of $(\gamma, \tau)$ [16]. Thus, clearly $\mathbf{D}(\mathcal{N})$ is a convex optimization with linear constraints. It consist of $O(B+M)$ variables and $O(B M)$ constraints. The objective function is the sum of $n$ terms, and the time required to evaluate the $i$ th term is $O\left(\left|\mathcal{B}_{i}\right|+\left|\mathcal{M}_{i}\right|\right)$, for any given value of the variables. Thus, the time required to obtain one member in $\mathcal{D}$, and hence one member of $\mathcal{O}$, is polynomial in $B, M, n$ [17]. Hence, the above theorem implies that, the time required to obtain one imputation that stabilizes the grand coalition, is polynomial in $B, M, n$.

Proof: Since $\mathcal{D} \neq \phi, \mathcal{O} \neq \phi$. We show that for an arbitrary $\mathbf{x}^{*} \in \mathcal{O}, \mathbf{x}^{*} \in \mathcal{C}$.

Consider an arbitrary $\mathrm{x}^{*} \in \mathcal{O}$, corresponding to some $\left(\boldsymbol{\lambda}^{*}, \boldsymbol{\beta}^{*}, \boldsymbol{\gamma}^{*}, \boldsymbol{\tau}^{*}\right) \in \mathcal{D}$. Now, $\sum_{i \in \mathcal{N}} x_{i}^{*}$ is the optimal value of the objective function of $\mathbf{D}(\mathcal{N})$. Since $\mathbf{D}(\mathcal{S})$ is the dual of $\mathbf{P}(\mathcal{S})$ for each $\mathcal{S} \subseteq \mathcal{N}$, by strong duality [16], and since $\mathbf{P}(\mathcal{N})$ is feasible, $\sum_{i \in \mathcal{N}} x_{i}^{*}=v(\mathcal{N})$. Now, we only need to show that $\sum_{i \in \mathcal{S}} x_{i}^{*} \geq v(\mathcal{S})$ for each $\mathcal{S} \subset \mathcal{N}$. If $\mathbf{P}(\mathcal{S})$ is infeasible, the result is immediate as $v(\mathcal{S})=-\infty$. Let $\mathbf{P}(\mathcal{S})$ be feasible. Then, by strong duality, $v(\mathcal{S})$ equals the optimum value of the objective function of $\mathbf{D}(\mathcal{S})$. The sub-vectors $\boldsymbol{\lambda}_{s}^{*}, \boldsymbol{\beta}_{s}^{*}, \boldsymbol{\gamma}_{s}^{*}, \boldsymbol{\tau}_{s}^{*}$ consisting of components of $\boldsymbol{\lambda}^{*}, \boldsymbol{\beta}^{*}, \boldsymbol{\gamma}^{*}, \boldsymbol{\tau}^{*}$ in $\mathcal{S}$, constitute a feasible solution of $\mathbf{D}(\mathcal{S})$, and $\sum_{i \in \mathcal{S}} x_{i}^{*}$ is the value of the objective function of $\mathbf{D}(\mathcal{S})$ for the above feasible solution. The optimum value of the objective function of $\mathbf{D}(\mathcal{S})$ is a lower bound for $\sum_{i \in \mathcal{S}} x_{i}^{*}$. Thus, we have shown that $\mathrm{x}^{*} \in \mathcal{C}$. The result follows.

Finally, we comment on the relation between Theorem 3.1 and some related literature in game theory. Samet et. al. [15] showed that when $v($.$) are the optimal$ values of the objective functions of linear programs, then the dual set of the linear program that generates $v(\mathcal{N})$, is a subset of the core. Markakis et. al. [14] showed that this result holds, when $v($.$) can be formulated as$ concave objective functions of a multicommodity flow problem. In our case, $v($.$) are the optimal values of$ the objective functions of concave optimizations. Note that the structure of the optimization we consider, is distinct from that of the multicommodity flow problem. Nevertheless, the proof of Theorem 3.1 uses the same techniques as those in the corresponding result in [14].

\section{B. Do other solution concepts stabilize the grand coali- tion?}

We next investigate the stability of two other well known solution concepts for sharing revenues in coalitional games: (i) nucleolus, (ii) Shapley value, and examine whether these belong to $\mathcal{C}$. The answer to this question determines whether these attain a socially desirable outcome by stabilizing the grand coalition.

We assume that $\mathbf{P}(\mathcal{S})$ is feasible, and hence $v(\mathcal{S})$ is finite, for all $\mathcal{S} \subseteq \mathcal{N}$.

We first consider the nucleolus.

Definition 3.3: The excess of a coalition $\mathcal{S}$ under an imputation $\mathbf{x}$ is $e_{S}(\mathbf{x})=v(\mathcal{S})-\sum_{i \in \mathcal{S}} x_{i}$; this is a measure of dissatisfaction of $\mathbf{S}$ under $\mathbf{x}$. Let $E(x)=$ $\left(e_{S}(\mathbf{x})\right)_{S \in 2^{\mathcal{N}}}$. The nucleolus is the set of imputations $x$ for which the vector $E(x)$ is lexicographically minimal.

In Example 2.1, the nucleolus is $((G+P) / 2,(3 G-$ P)/2).

The nucleolus of any coalitional game is a singleton ( $\mathrm{p}$. 288 , [2]). Whenever the core of a coalitional game is non-empty, the nucleolus belongs to the core. This is because, for any imputation $x$ in the core, $E(x) \leq 0$, i.e., the maximum excess is negative. Hence for any imputation $x^{*}$, which leads to lexicographically minimal excess vector among all the imputations, the corresponding maximum excess will be negative. Hence, $E\left(x^{*}\right) \leq 0$. Thus, from (1), $x^{*}$ belongs to the core.

Thus, owing to Theorem 3.1, the nucleolus belongs to $\mathcal{C}$, and hence renders the grand coalition stable. 
We next consider the Shapley value.

Definition 3.4: For any $i$, and $\mathcal{S} \subset \mathcal{N}$ such that $i \notin \mathcal{S}$, let $\Delta_{i}(\mathcal{S})=v(\mathcal{S} \cup\{i\})-v(\mathcal{S})$. The Shapley value is the imputation $\mathbf{x}$ for which

$$
x_{i}=\frac{1}{n !} \sum_{U \in \mathcal{U}} \Delta_{i}\left(\mathcal{S}_{i}(U)\right),
$$

where $\mathcal{U}$ is the set of all orderings of the set of players, and $\mathcal{S}_{i}(U)$ is the set of players preceding $i$ in ordering $U$.

In Example 2.1, $\Delta_{i}(\phi)=v(\{i\}), \Delta_{1}(2)=G, \Delta_{2}(1)=$ $2 G-P$, and the Shapley value is $((G+P) / 2,(3 G-$ $P) / 2)$.

The significance of the Shapley value is that it is the unique imputation that attains the following properties (p. 292, [2]). (a) If $i$ and $j$ are interchangeable, i.e., $\Delta_{i}(\mathcal{S})=\Delta_{j}(\mathcal{S})$ for each $\mathcal{S}$ such that $i, j \notin \mathcal{S}$, then the imputation allocates equal revenues to both $i$ and $j$ (symmetry). (b) If $\Delta_{i}(\mathcal{S})=v(\{i\})$ for each $\mathcal{S}$ such that $i \notin \mathcal{S}$, then the imputation allocates revenue $v(\{i\})$ to $i$ (dummy player allocation). (c) Consider two coalitional games with the set of players $\mathcal{N}$, and the characteristic functions $w_{1}(),. w_{2}($.$) , and a third coalitional game with$ the same set of players, and the characteristic function $w_{1}()+.w_{2}($.$) . Then, the imputation that constitutes the$ Shapley value for the third coalitional game equals the sum of those for the first two (additivity).

We next demonstrate that the Shapley value need not be in $\mathcal{C}$.

Lemma 3.1: For $n=2$, the Shapley value is in $\mathcal{C}$. For $n>2$, the Shapley value need not be in $\mathcal{C}$.

Proof: First, let $n=2$. Let $\left(x_{1}, x_{2}\right)$ be the Shapley value. We need to show that $x_{i} \geq v(\{i\})$ for $i=1,2$. Now, since $v(\mathcal{S})$ is finite for any $\mathcal{S} \subseteq \mathcal{N}$, and the game is superadditive, $\Delta_{i}(\mathcal{S}) \geq v(\{i\})$, for any $i$ and $\mathcal{S} \subset \mathcal{N}$ such that $i \notin \mathcal{S}$. The result follows from (2).

Now, let $n=3$. Consider a system with (i) $\mathcal{B}_{i}=\{i\}$ $\mathcal{M}_{i}=\{i\}$, for each operator $i$, (ii) $U_{i}\left(\mathbf{y}^{i}\right)=y_{i}$, and (iii) $m_{k}=0$ for each $k \in \mathcal{M}$. Let $R_{12}=R_{21}=R_{23}=$ $R_{32}=1$ and $R_{j k}=0$ otherwise. Refer to Figure 1 .

Clearly, $v(\{i\})=0 \forall i, v(\{1,2\})=v(\{2,3\})=$ $v(\{1,2,3\})=2, v(\{1,3\})=0$. Table I shows all possible orderings $U$ of the operators, and $\Delta_{i}(U)$ for each operator $i$ and ordering $U$. From (2) and Table I, the Shapley value of the operators is $\mathbf{x}=\left(\frac{2}{6}, \frac{8}{6}, \frac{2}{6}\right)$. Note that $x_{1}+x_{2}=\frac{10}{6}<v(\{1,2\})$. Hence $\mathbf{x} \notin \mathcal{C}$.

Thus, the Shapley value does not in general render the grand coalition stable for the class of games we consider, and hence is not a desirable basis for allocating the revenues in these games.

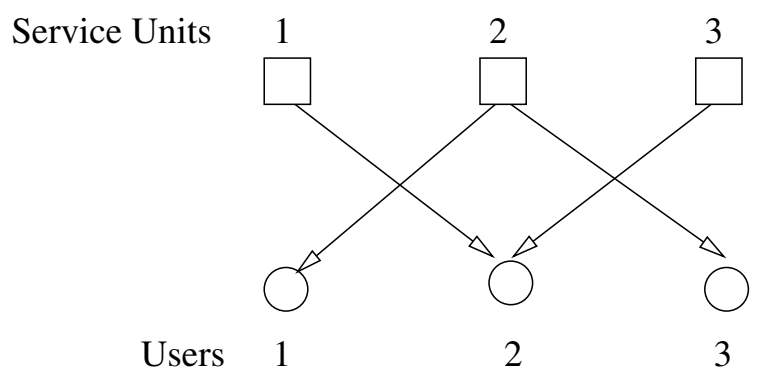

Fig. 1. Links between the service units and the users that provide non-zero rates. Each of the shown links has rate 1.

TABLE I

ALL POSSIBLE ORDERINGS AND MARGINAL CONTRIBUTIONS OF THE PLAYERS.

\begin{tabular}{c|c|c|c}
$U$ & $\Delta_{1}(U)$ & $\Delta_{2}(U)$ & $\Delta_{3}(U)$ \\
\hline 123 & 0 & 2 & 0 \\
132 & 0 & 2 & 0 \\
213 & 2 & 0 & 0 \\
231 & 0 & 0 & 2 \\
312 & 0 & 2 & 0 \\
321 & 0 & 2 & 0
\end{tabular}

Finally, note that the proof for Lemma 3.1 shows that the Shapley value is not in the core even when (i) each operator owns one service unit and one user each, (ii) the utility functions are linear, and (iii) the users do not require any minimum rate guarantees. In this special case, the coalitional game we consider, becomes the same as the well known permutation game [18], [19]. Thus, our proof reveals that the Shapley value does not belong to the core for permutation games.

\section{Optimal selection of SLAs using different solution concepts}

Example 2.1 has demonstrated that a coalition can induce unfairness for the users, and can deteriorate the throughput attained by some of them. The users therefore negotiate SLAs (here, the minimum rate guarantees) with the operators apriori. We now demonstrate, how the operators can use the above solution concepts to determine which SLAs they ought to accept. The SLAs impose the minimum rate constraints in $\mathbf{P}(\mathcal{S})$ for the coalitions $\mathcal{S}$, and hence may reduce the aggregate revenues $v($.$) generated by the coalitions. But, the main$ consideration for an operator is whether the SLAs reduce the revenue it earns. Also, an operator may be able to honor some SLAs even when it does not cooperate with other operators, and some other SLAs only when it cooperates with others. The question therefore is whether an operator ought to accept all SLAs that it can honor by itself, or should also accept some SLAs it can honor only in coalitions. Using simple variations in Example 2.1, we 
show that the answers to the above questions can often be counter-intuitive.

In Example 2.1, recall that $\mathcal{C}=\left\{\mathbf{x} \in \mathbb{R}^{2}: x_{1}+x_{2}=\right.$ $\left.2 G, x_{1} \geq P, x_{2} \geq G\right\}$. Thus, $\min _{\mathbf{x} \in \mathcal{C}} x_{1}=P$, and hence operator 1 earns a revenue of at least $P$, as long as the imputation is in $\mathcal{C}$. Also, the nucleolus is $((G+P) / 2,(3 G-P) / 2)$. When the operators cooperate, the users of operator 1 do not receive any service, i.e., have 0 rates, and therefore generate 0 revenue. Now, suppose they demand minimum rate guarantees of $z>0$ each, and suppose operator 1 does not agree to these demands, and these users subsequently leave. Then, $\mathcal{M}_{1}=\phi$, and $v(\{1\})=0$. But, the function $v($.$) does not change otherwise. Thus, \mathcal{C}=\left\{\mathbf{x} \in \mathbb{R}^{2}\right.$ : $\left.x_{1}+x_{2}=2 G, x_{1} \geq 0, x_{2} \geq G\right\}, \min _{\mathbf{x} \in \mathcal{C}} x_{1}=0$, and the nucleolus is $(G / 2,3 G / 2)$. Thus, operator 1 may not earn any revenue even when the imputation is in the core, and the nucleolus also gives it less revenue than in the previous case. Thus, although the users of an operator do not generate any revenue, their mere presence may enhance the revenues earned by the operator. Thus, the operators need to judiciously determine, which SLAs to accept.

Now, assume that operator 1 agrees to provide minimum rates $m_{1}$ and $m_{2}$ to its users, where $m_{1}+m_{2} \leq P$. Thus, $\mathbf{P}(\mathcal{S})$ is feasible for each $\mathcal{S} \subseteq \mathcal{N}$. Now, $v(\{1,2\})=$ $m_{1}+m_{2}+\left(2-\frac{m_{1}+m_{2}}{P}\right) G$ and $v($.$) function is unchanged$ otherwise. Thus, $\mathcal{C}=\left\{\mathbf{x} \in \mathbb{R}^{2}: x_{1}+x_{2}=m_{1}+m_{2}+\right.$ $\left.\left(2-\frac{m_{1}+m_{2}}{P}\right) G, x_{1} \geq P, x_{2} \geq G\right\}, \min _{\mathbf{x} \in \mathcal{C}} x_{1}=P$, and the nucleolus is $\left(\frac{P+m_{1}+m_{2}}{2}+\left(1-\frac{m_{1}+m_{2}}{P}\right) G / 2,(3-\right.$ $\left.\left.\frac{m_{1}+m_{2}}{P}\right) G / 2-\frac{P-m_{1}-m_{2}}{2}\right)$. Thus, the minimum revenue earned by operator 1 exceeds that when operator 1 does not accept the SLAs (and subsequently lose both of its users), when the imputation is in the core, . Thus, if operator 1 always earns this minimum revenue, it should accept the SLAs. When the imputation is the nucleolus, even then, the revenue earned by operator 1 exceeds that when the SLAs are not accepted, provided $m_{1}+m_{2}<$ $P^{2} /(G-P)$. Thus, if operator 1 either needs to accept both the SLAs or reject both the SLAs, it should select the first option, provided $m_{1}+m_{2}<P^{2} /(G-P)$, and should select the second otherwise.

Next, suppose that operator 1 accepts the SLA of user $k$, and rejects that of the other user. Clearly, $m_{k} \leq$ $m_{1}+m_{2} \leq P$. Still, $\mathbf{P}(\mathcal{S})$ is feasible for each $\mathcal{S} \subseteq \mathcal{N}$. Now, $v(\{1,2\})=m_{k}+\left(2-\frac{m_{k}}{P}\right) G$, and $v($.$) function is$ unchanged otherwise. Thus, $\mathcal{C}=\left\{\mathbf{x} \in \mathbb{R}^{2}: x_{1}+x_{2}=\right.$ $\left.m_{k}+\left(2-\frac{m_{k}}{P}\right) G, x_{1} \geq P, x_{2} \geq G\right\}, \min _{\mathbf{x} \in \mathcal{C}} x_{1}=P$, and the nucleolus is $\left(\frac{P+m_{k}}{2}+\left(1-\frac{m_{k}}{P}\right) G / 2,\left(3-\frac{m_{k}}{P}\right) G / 2-\right.$ $\left.\frac{P-m_{k}}{2}\right)$. Thus, if operator 1 always earns the minimum revenue, subject to the imputation being in the core, it should accept at least one SLA - its revenue remains same irrespective of whether it accepts one or both the SLAs. On the other hand, if the revenues are allocated as per the nucleolus, then operator 1 earns greater revenue, if it accepts one SLA rather than accepting both SLAs. If it accepts one SLA, it should accept the less stringent SLA (i.e., the SLA which prescribes a lower value of the minimum rate). Furthermore, it should accept at least one SLA if and only if $\min \left(m_{1}, m_{2}\right)<P^{2} /(G-P)$, and should reject both SLAs if $\min \left(m_{1}, m_{2}\right) \geq P^{2} /(G-$ $P)$. Summarily, if the revenue allocation is as per the nucleolus, operator 1 should accept the less stringent $S L A$, provided $\min \left(m_{1}, m_{2}\right)<P^{2} /(G-P)$, and reject both otherwise. When $P<G / 3, P^{2} /(G-P)<P / 2$, then there exist $m_{1}, m_{2}$ such that $P^{2} /(G-P)<$ $\min \left(m_{1}, m_{2}\right)<P / 2$ and $m_{1}+m_{2}<P$. For any such $\left(m_{1}, m_{2}\right)$, operator 1 rejects both the SLAs, although it can satisfy both together even when it does not form a coalition with operator 2. Thus, an operator may reject all the SLAs even when it can satisfy all of them simultaneously by itself.

Next, we consider the situation in which the minimum rate requirements are such that operator 1 cannot satisfy them by itself, but can satisfy if forms a coalition with operator 2. Thus $v(\{1\})=-\infty$. Since users of operator 2 do not have any minimum rate requests, $\mathbf{P}(\{2\})$ is feasible, and $\mathbf{P}(\{1,2\})$ is also feasible by assumption. Thus, $\mathcal{C}=\left\{\mathbf{x} \in \mathbb{R}^{2}: x_{1}+x_{2}=v(\{1,2\}), x_{2} \geq G\right\}$. Thus, $\inf _{\mathbf{x} \in \mathcal{C}} x_{1}=-\infty$, and operator 2 can compel operator 1 to pay it any amount it deems fit, even when the revenue allocation is in the core. Thus, in this case operator 1 should not agree to such SLAs. Note that the nucleolus is ill-defined in this case.

We now consider a different game, to examine whether an operator should always refuse SLAs that it can not satisfy by itself. Let $n=3, \mathcal{B}_{i}=\{i\}, \mathcal{M}_{i}=\{i\}, i=$ $1,2,3$. Let $R_{1 k}=G, k=2,3, R_{j 1}=G, j=2,3$, where $G>0$, and $R_{j k}=0$, otherwise. Also, let $m_{1}>0, m_{k}=0$ for $k>1$. Assume linear utility functions, i.e., $U_{i}\left(\mathbf{y}^{i}\right)=y_{i}$ for each $i$. Since $R_{11}=0$, operator 1 cannot provide rate $m_{1}$ to user 1 unless it forms a coalition with another operator. Thus, $v(\{1\})=$ $-\infty, v(\{i\})=0, i>1, v(\{1,2\}=v(\{1,3\})=$ $v(\{1,2,3\})=2 G, v(\{2,3\})=0$. Thus, $\mathcal{C}=\{\mathbf{x} \in$ $\mathbb{R}^{3}: x_{1}+x_{2}+x_{3}=2 G, x_{1}+x_{2} \geq 2 G, x_{1}+x_{3} \geq$ $\left.2 G, x_{2} \geq 0, x_{3} \geq 0\right\}$. If $\mathbf{x} \in \mathcal{C}, x_{1}+x_{2}=2 G-x_{3}$. Thus, $2 G \leq 2 G-x_{3}$, which implies that $x_{3} \leq 0$. Since $x_{3} \geq 0$, $x_{3}=0$. Similarly, it can be shown that $x_{2}=0$. Hence, $x_{1}=2 G-x_{2}-x_{3}=2 G$. Thus, $\mathcal{C}=\{(2 G, 0,0)\}$. Now, if operator 1 refuses the SLA of user $1, \mathcal{M}_{1}=\phi$, $v(\{i\})=0, \forall i, v(\{1,2\}=v(\{1,3\})=v(\{1,2,3\})=$ $G, v(\{2,3\})=0$. Thus, $\mathcal{C}=\left\{\mathbf{x} \in \mathbb{R}^{3}: x_{1}+x_{2}+x_{3}=\right.$ $\left.G, x_{1}+x_{2} \geq G, x_{1}+x_{3} \geq G, x_{i} \geq 0, \forall i\right\}$. Arguing as before, $\mathcal{C}=\{(G, 0,0)\}$. Thus, the revenue of operator 1 
decreases when it refuses the SLA of its user. Hence, an operator's revenue may increase when it accepts a SLA it can not satisfy by itself, and so it may register an user whose SLA, it can not satisfy by itself.

\section{Selecting an imputation in the core}

We now examine how the revenue allocations can be selected amongst the imputations in $\mathcal{C}$. The question is relevant as $\mathcal{C}$ usually consists of multiple imputations, e.g., as in Example 2.1. One possibility is to select an imputation in $\mathcal{C}$ that maximizes a concave objective function. Once the objective is determined, the optimal imputation can be obtained using the characterization of $\mathcal{C}$ in (1). But, since (1) characterizes $\mathcal{C}$ using $2^{n}$ inequalities, the computation time for such an imputation will be exponential in $n$. We now examine whether $\mathcal{C}$ can be characterized using polynomial number of convex inequalities, which will in turn ensure that the computation time for such imputations will be polynomial in $n, B, M$. Note that this is unlikely in several well-known coalitional games, as a simpler question of determining whether a given imputation is in the core turns out to be NP-hard in these games [20]. In our context, we answer this question only in part.

We show that $\mathcal{C}$ can be characterized using polynomial number of inequalities in the special case that (i) each operator owns at most one service unit and at most one user, (ii) the utility functions are linear, and (iii) the users do not require minimum rate guarantees. This case can be reduced to the case that each operator owns exactly one service unit and one user, by introducing dummy service units $j$ (users $k$ ) as required, with $R_{j k}=0$ for all such $j(k)$. Note that the modified game has the same set of players and characteristic function as the original game, and therefore has the same core. The modified game has the same form as permutation game, and $\mathcal{C}=$ $\mathcal{O}$ in this game [19]. Note that in this special case $\mathcal{O}$ can be characterized as follows.

$$
\begin{array}{r}
\mathcal{O}=\left\{\mathbf{x} \in \mathbb{R}^{n}: x_{i}=\sum_{j \in \mathcal{B}_{i}} \lambda_{j}+\sum_{k \in \mathcal{M}_{i}} \beta_{k}, \sum_{i \in \mathcal{N}} x_{i}=v(\mathcal{N}),\right. \\
\left.\lambda_{j}+\beta_{k} \geq R_{j k}, \lambda_{j} \geq 0, \beta_{k} \geq 0, \forall j \in \mathcal{B}, k \in \mathcal{M}\right\}
\end{array}
$$

Thus, $\mathcal{C}$ can be characterized using $O(n+B+M)$ variables and $O(B M)$ constraints.

Using a counter example, we next show that $\mathcal{O}$ is in general a proper subset of $\mathcal{C}$ when (i) does not hold even if (ii) and (iii) hold. This is an important distinction between the game we consider and the permutation game. The example also shows that (a) an imputation $\mathbf{x}$ that is maxmin fair in $\mathcal{C}$, and maximizes $\sum_{i \in \mathcal{N}} \log x_{i}$ in $\mathcal{C}$, and (b) the nucleolus, need not belong to $\mathcal{O}$. Thus, $\mathcal{O}$ may not consider several important imputations in $\mathcal{C}$.
Lemma 3.2: There exists a system where (i) $n=2$, (ii) $\left|\mathcal{B}_{i}\right|=1$ for each operator $i,\left|\mathcal{M}_{i}\right|>1$ for some $i$, (iii) the utility functions are linear, and (iv) the users do not require minimum rate guarantees, and $\mathcal{O} \subset$ $\mathcal{C}$. Furthermore, in this system, the nucleolus and the imputations $\mathbf{x}$ that are maxmin fair in $\mathcal{C}$ and maximize $\sum_{i \in \mathcal{N}} \log x_{i}$ in $\mathcal{C}$, do not belong to $\mathcal{O}$.

Proof: Consider a system with (i) $n=2$, (ii) $\left|\mathcal{B}_{i}\right|=$ 1 for each operator $i,\left|\mathcal{M}_{1}\right|=m, \mathcal{M}_{2}=\phi$ (iii) $U_{i}\left(\mathbf{y}^{i}\right)=$ $\sum_{k \in \mathcal{M}_{i}} y_{k}$, and (iv) $m_{k}=0$ for each $k \in \mathcal{M}$. Since operator 2 does not have any user, we do not specify its utility function. Let $R_{1 k}=P, R_{2 k}=G$ for each $k \in \mathcal{M}$, where $G>P>0$. Thus $(G+P) / 2>P>0$. Clearly, $v(\{1\})=P, v(\{2\})=0, v(\{1,2\})=G+P$. Consider the imputation $\mathbf{x}=((G+P) / 2,(G+P) / 2)$. From (1), and since $(G+P) / 2>P>0, \mathbf{x} \in \mathcal{C}$. Also, $\mathbf{x}$ is maxmin fair in $\mathcal{C}$, and maximizes $\log \left(z_{1}\right)+\log \left(z_{2}\right)$ among all $\mathbf{z} \in \mathcal{C}$. We show that $\mathbf{x} \notin \mathcal{O}$ when $m$ is sufficiently large.

In this case, $\mathbf{D}(\{1,2\})$ is the following linear program.

$$
\begin{array}{rr}
\min \lambda_{1}+\lambda_{2}+\sum_{k=1}^{m} \beta_{k} & \\
\text { s.t. } \lambda_{1}+\beta_{k} \geq P, & 1 \leq k \leq m \\
\lambda_{2}+\beta_{k} \geq G, & 1 \leq k \leq m \\
\lambda_{1} \geq 0, \lambda_{2} \geq 0, \beta_{k} \geq 0, & 1 \leq k \leq m
\end{array}
$$

Suppose $\mathbf{x} \in \mathcal{O}$. Then,

$$
\begin{aligned}
\lambda_{1}^{*}+\sum_{k=1}^{m} \beta_{k}^{*} & =\frac{G+P}{2} \\
\lambda_{2}^{*} & =\frac{G+P}{2}
\end{aligned}
$$

for some optimal solution $\left(\boldsymbol{\lambda}^{*}, \boldsymbol{\beta}^{*}\right)$ of $\mathbf{D}(\{1,2\})$. Thus, from (4) and (7),

$$
\beta_{k}^{*} \geq G-\frac{G+P}{2}=\frac{G-P}{2}>0,1 \leq k \leq m
$$

Thus, from (5),

$$
\lambda_{1}^{*}+\sum_{k=1}^{m} \beta_{k}^{*} \geq m\left(\frac{G-P}{2}\right)>\frac{G+P}{2} \text { for large } m
$$

This contradicts (6). The result follows.

The nucleolus is $\mathbf{w}=(P+G / 2, G / 2)$ in this case. We show that $\mathbf{w} \notin \mathcal{O}$ for sufficiently large $m$. Suppose $\mathbf{w} \in$ $\mathcal{O}$. Then, again, $\lambda_{1}^{*}+\sum_{k=1}^{m} \beta_{k}^{*}=G / 2+P$ and $\lambda_{2}^{*}=G / 2$ for some optimal solution $\left(\boldsymbol{\lambda}^{*}, \boldsymbol{\beta}^{*}\right)$ of $\mathbf{D}(\{1,2\})$. Thus, from (4), $\beta_{k}^{*} \geq G-G / 2=G / 2>0,1 \leq k \leq m$. Thus, from (5), $\lambda_{1}^{*}+\sum_{k=1}^{m} \beta_{k}^{*} \geq m G / 2>(G / 2+P)$ for large enough $m$. This contradicts the fact that $\lambda_{1}^{*}+\sum_{k=1}^{m} \beta_{k}^{*}=$ $G / 2+P$. The result follows. 
This suggests that, it is in general unlikely that $\mathcal{C}$ can be characterized by polynomial number of inequalities for the class of games we consider.

\section{Generalization of the COALITIONAL GAME MODEL IN PRESENCE OF PRIOR PREFERENCES}

We have shown that if an operator decides which coalition to join based only on the revenue it can earn thereby, it will be possible to induce each operator to join the grand coalition (Section III-A). But, the operators may make such decisions based on additional considerations such as prior trust relations. We now generalize the coalitional game model and our analytical results to this case.

Now, each operator decides apriori which other operators it can cooperate with, and decides which ones among these it would actually cooperate with, based on the revenue considerations. We also assume that (a) if $i$ can cooperate with $j, j$ can also cooperate with $i$, and (b) $i$ can cooperate with $j$ if there exists operators $k_{1}, \ldots, k_{p}$, such that $i$ can cooperate with $k_{1}, k_{l}$ can cooperate with $k_{l+1}$ for $l=1, \ldots, p-1$, and $k_{p}$ can cooperate with $j$. The motivation behind (a) is that trust relations are oftentimes mutual. The motivation behind (b) is that entities trust each other if they trust a common entity. This scenario can be modeled by representing the operators as vertices of an undirected graph, and allowing an edge to exist between a pair of vertices if and only if the operators can cooperate. The resulting graph consists of components that are cliques. Let the number of components be $K$, and the components be $\mathcal{N}_{1}, \ldots, \mathcal{N}_{K}$. Now, $v(\mathcal{S})$ is defined as before if $\mathcal{S}$ is a subset of such a component. Otherwise, $v(\mathcal{S})=\sum_{k=1}^{K} v\left(\mathcal{S} \cap \mathcal{N}_{k}\right)$. Thus $v(\mathcal{S})$ is obtained by concatenating the solutions of $\mathbf{P}\left(\mathcal{S} \cap \mathcal{N}_{k}\right)$. Thus, if operators $l, r$ are in different components of the above graph, $\alpha_{j k}=0$ if $j \in \mathcal{B}_{l}$, $k \in \mathcal{M}_{r}$, even when $l, r$ are in a coalition. Thus, such operators never use each others' resources, and never cooperate physically. So, each coalition $\mathcal{S}$ effectively consists of disjoint coalitions $\mathcal{S} \cap \mathcal{N}_{1}, \ldots, \mathcal{S} \cap \mathcal{N}_{K}$, and when an operator in the component $k$ joins a coalition $\mathcal{S}$, it effectively joins a coalition of operators that are in $\mathcal{S} \cap \mathcal{N}_{k}$.

Let $\mathcal{C}^{\prime}$ be the core of this game. Let $\mathcal{O}_{k}$ be defined for $\mathbf{D}\left(\mathcal{N}_{k}\right)$, as $\mathcal{O}$ was defined for $\mathbf{D}(\mathcal{N})$. Let

$$
\mathcal{O}^{\prime}=\mathcal{O}_{1} \times \ldots \times \mathcal{O}_{K}
$$

Theorem 4.1: Let $\mathbf{P}\left(\mathcal{N}_{k}\right)$ be feasible for $k=1, \ldots, K$. Then, $\mathcal{O}^{\prime} \neq \phi$ and $\mathcal{O}^{\prime} \subseteq \mathcal{C}^{\prime}$.

The proof is similar to that for Theorem 3.1, and is omitted for brevity. Thus, an imputation in the core can be obtained by solving $K$ concave optimizations, each with $O(B+M)$ variables and $O(B M)$ constraints.

Next, as in Section III-B, since $\mathcal{C}^{\prime} \neq \phi$, the nucleolus belongs to $\mathcal{C}^{\prime}$, and hence stabilizes the grand coalition. Lemma 3.1 holds in this case well - the Shapley value does not in general ensure that the grand coalition is stable.

Finally, a desirable imputation in $\mathcal{C}^{\prime}$ can be computed solving an optimization problem, as described in Section III-D. Towards this end, using $v($.$) functions as$ described in this section, $\mathcal{C}^{\prime}$ can be characterized using $2^{n}$ linear equalities, as $\mathcal{C}$ was characterized by (1). But, the time required to compute such imputations depends on the number of inequalities required to characterize $\mathcal{C}^{\prime}$. We next show that such a characterization can be accomplished using $\sum_{k=1}^{K} 2^{\left|\mathcal{N}_{k}\right|}$ linear equalities.

A coalition $\mathcal{S}$ is said to be essential if for any proper partition $\mathcal{P}$ of $\mathcal{S}, v(\mathcal{S})>\sum_{\mathcal{T} \in \mathcal{P}} v(\mathcal{T})$. Let $\mathcal{E}$ be the set of essential coalitions. It is well known that the core can be completely characterized using only the essential coalitions [21]. This follows because for any imputation $\mathbf{x}, \sum_{i \in \mathcal{S}} x_{i} \geq v(\mathcal{S})$ for each coalition $\mathcal{S}$ if and only if this holds for all essential coalitions. Also, since $v(\mathcal{N})=\sum_{k=1}^{K} v\left(\mathcal{N}_{k}\right)$ in this case, when $\sum_{i \in \mathcal{S}} x_{i} \geq v(\mathcal{S})$ for each coalition $\mathcal{S}, \sum_{i \in \mathcal{N}} x_{i}=v(\mathcal{N})$ if and only if $\sum_{i \in \mathcal{N}_{k}} x_{i}=v\left(\mathcal{N}_{k}\right)$ for each $k=1, \ldots, K$. Thus,

$$
\begin{aligned}
\mathcal{C}^{\prime}=\left\{\mathrm{x} \in \mathbb{R}^{n}:\right. & \sum_{i \in \mathcal{N}_{k}} x_{i}=v\left(\mathcal{N}_{k}\right), 1 \leq k \leq K \\
& \left.\sum_{i \in \mathcal{T}} x_{i} \geq v(\mathcal{T}), \forall \mathcal{T} \in \mathcal{E}\right\}
\end{aligned}
$$

We show that the number of essential coalitions is at most $\sum_{k=1}^{K} 2^{\left|\mathcal{N}_{k}\right|}$ in this case. Let a coalition $\mathcal{S}$ consist of operators in multiple components $\mathcal{N}_{k}$ s. Then, consider the partition $\mathcal{P}$ consisting of $\mathcal{S} \cap \mathcal{N}_{k}$ for $k=1, \ldots K$. Clearly, $\mathcal{P}$ is a proper partition of $\mathcal{S}$. Also, $v(\mathcal{S})=$ $\sum_{\mathcal{T} \in \mathcal{P}} v(\mathcal{T})$. Thus, $\mathcal{S}$ is not an essential coalition. Thus, only the subsets of the components can be in $\mathcal{E}$, and there are only $\sum_{k=1}^{K} 2^{\left|\mathcal{N}_{k}\right|}$ such subsets. Now, the result follows from (8).

Note that $\sum_{k=1}^{K} 2^{\left|\mathcal{N}_{k}\right|} \leq 2^{n}$, and the difference between the two may be substantial. For example, when $\max _{k}\left|\mathcal{N}_{k}\right|$ (i.e., the maximum number of operators who are willing to cooperate with each other) is $O(\log n)$, then $\sum_{k=1}^{K} 2^{\left|\mathcal{N}_{k}\right|}$ is a polynomial in $n$.

\section{CONCLUSION}

We have considered the scenario in which several operators in a geographical area offer downlink wireless data access to subscribers. Operators can cooperate, thereby 
pooling their network resources, and agreeing to serve each other's subscribers. We have taken the aggregate revenue earned by a coalition to be the maximum sum utility over the cooperating operators, subject to the various physical rate constraints between the subscribers and the various operators. Such a formulation leads to a coalitional game. The characteristic function is obtained as the value of an optimization problem with a concave objective function and linear constraints. In Theorem 3.1 we have shown that this coalitional game has a nonempty core. In particular, the dual of a nonlinear program provides a subset, $\mathcal{O}$, of the core. We have also investigated the nucleolus and the Shapely value of such coalition games. The nucleolus and certain interesting imputations, defined in terms of optimization problems, need not be in the set $\mathcal{O}$ (see Lemma 3.2). Whenever the core is non-empty, the nucleolus is in the core, but in general, the Shapely value will not be in the core (see Lemma 3.1). We found, however, that a coalition that increases total revenue can induce unfairness for the users of some of the operators and may therefore reduce the customer base of these operators. Due to the possibility of unfairness, it was interesting to consider minimum rate agreements (or service level agreements, SLAs) between operators and subscribers. Our investigations reveal that the operators need to judiciously determine which SLAs to accept. We have also investigated criteria for selecting a particular member of the core, when the core is nonempty. Finally, we have shown that the grand coalition is stabilizable even when the operators cooperate not merely looking at the revenues, but also taking into account additional considerations such as prior trust relations (Theorem 4.1).

\section{ACKNOWLEDGMENT}

We would like to thank Eitan Altman for introducing us to the techniques of coalitional games. We also thank Alireza Aaram for suggestions regarding utility functions.

\section{REFERENCES}

[1] T. A. Weiss and F. K. Jondral, "Spectrum pooling: an innovative strategy for the enhancement of spectrum efficiency," IEEE Communications Magazine, vol. 42, no. 3, pp. S8-14, March 2004.

[2] M. Osborne and A. Rubinstein, A Course in Game Theory. Cambridge, PA: The MIT press, 1999.

[3] M. M. Halldorsson, L. Li, J. Y. Halpern, and V. S. Mirrokni, “On spectrum sharing games," in Proceedings of PODC, St. Johns, Newfoundland, Canada, July, 25-28 2004.

[4] R. Etkin, A. Parekh, and D. Tse, "Spectrum sharing for unlicensed bands," IEEE Journal on Selected Areas in Communications, vol. 25, no. 3, pp. 517-528, April 2007.

[5] E. Altman, V. S. Borkar, A. A. Kherani, P. Michiardi, and R. Molva, "Some game-theoretic problems in wireless ad-hoc networks," in EuroNGI Workshop, 2004, pp. 82-104.
[6] S. Shakkotai, E. Altman, and A. Kumar, "Multihoming of users to access points in wlans: A population game perspective," IEEE Journal on Selected Areas in Communications, Special Issue on Noncooperative Behaviour in Networking, vol. 25, no. 6, pp. 1207-1215, August 2007.

[7] M. Felegyhazi and J.-P. Hubaux, "Wireless operators in a shared spectrum," ACM SIGMOBILE Mobile Computing and Communications Review, vol. 10, no. 4, pp. 13-14, October 2006.

[8] J. E. Suris, L. A. DaSilva, Z. Han, and A. B. MacKenzie, "Cooperative game theory for distributed spectrum sharing," in Proceedings of ICC, 2007.

[9] Z. Han and H. V. Poor, "Coalitional games with cooperative transmission: A cure for the curse of boundary nodes in selfish packet-forwarding wireless networks," in Proceedings of 5th International Symposium on Modeling and Optimization in Mobile, Ad Hoc, and Wireless Networks, 2007.

[10] S. Mathur, L. Sankaranarayanan, and N. Mandayam, "Coalitional games in cooperative radio networks," in Fortieth Asilomar Conference on Signals, Systems and Computers, Oct.-Nov. 2006, pp. 1927-1931.

[11] V. Conitzer and T. Sandholm, "Complexity of determining nonemptiness of the core," Technical Reprt, CS-02-137, CMU, 2002.

[12] L. S. Shapley and M. Shubik, "The assignment game i: The core," International Journal of Game Theory 1, pp. 111-130, 1972.

[13] J. Sanchez-Soriano, M. A. Lopez, and I. Garcia-Jurado, "On the core of transportation games," Mathematical Social Sciences, vol. 41, pp. 215-225, 2001.

[14] E. Markakis and A. Saberi, "On the core of the multicommodity flow game," Decision support systems, vol. 39, pp. 3-10, 2005.

[15] D. Samet and E. Zamel, "On the core and dual set of linear programming games," Mathematics of Operations Research, vol. 9, no. 2, pp. 309-316, May 1984.

[16] S. Boyd and L. Vandenberghe, Convex Optimization. Cambridge University Press, 2004.

[17] S. M. Stefanov, "Convex separable minimization problems with a linear constraint and bounded variables," International Journal of Mathematics and Mathematical Sciences, vol. 2005, no. 9, pp. 1339-1363, 2005.

[18] I. Curiel and S. Tijs, "Assignment games and permutation games," Methods of Operations Research, vol. 54, pp. 323-334, 1986.

[19] T. Quint, "On one-sided versus two-sided matching games," Games and Economic Behavior, vol. 16, no. 1, pp. 124-334, September 1996.

[20] Q. Feng, S. Zhu, M. Cai, and X. Deng, "Membership for core of lp games and other games," Lecture notes on computer science, vol. 2108, pp. 247-256, 2001, springer Verlag, Berlin Heidelberg.

[21] G. Huberman, "The nucleolus and the essential coalitions," in Analysis and Optimization of Systems. Springer Berlin/Heidelberg, 1980, pp. 416-422. 